\title{
A perspectiva do estudante de graduação em Odontologia na capacitação de Agentes Comunitários de Saúde
}

\author{
Camila Marina*; Egídio Antonio Demarco**; Patrícia Távora Bulgarelli***; Alexandre Favero \\ Bulgarelli****
}

\author{
Cirurgiã-dentista, Faculdade de Odontologia da \\ Universidade Federal do Rio Grande do Sul \\ ** Mestre, docente da Escola de Saúde Comunitária do \\ Grupo Hospitalar Conceição \\ *** Mestranda em Saúde Coletiva, Escola de Enferma- \\ gem da Universidade Federal do Rio Grande do Sul \\ Doutor, docente de Saúde Coletiva, Faculdade de \\ Odontologia da Universidade Federal do Rio Grande \\ do Sul
}

\section{RESUMO}

$\mathrm{Na}$ atualidade brasileira, as Diretrizes Curriculares Nacionais para os cursos de Odontologia recomendam ofertar oportunidades distintas, em diversos cenários de aprendizado, para formação de futuros cirurgiões-dentistas com percepções holísticas e integradoras do cuidado em saúde. $\mathrm{O}$ presente artigo apresenta um relato de experiência de um estudante de graduação na construção de uma capacitação de agentes comunitários de saúde sobre o tema cuidados em saúde bucal. A referida ação de capacitação foi fundamental no processo de aprendizagem do aluno e para os profissionais da saúde na Estratégia de Saúde da Família onde foi realizada.

Descritores: Educação em Saúde. Saúde Pública. Sistema Único de Saúde.

\section{INTRODUÇÃO}

Uma vez estabelecida a Atenção Primaria em Saúde (APS) como o eixo coordenador das ações do Sistema Único de Saúde (SUS), a partir de 1991, o Ministério da Saúde (MS) vem incentivando e proporcionando a atuação de Agentes
Comunitários de Saúde (ACS) em ações de promoção da saúde e prevenção das doenças ${ }^{1}$. Os ACS reforçam o vínculo entre a comunidade e o sistema de saúde, contribuindo para maior efetividade das ações de promoção da saúde, prevenção das doenças e assistência individual. As características do trabalho dos ACS, bem como os efeitos positivos de sua atuação mostram-se relevantes na efetividade de cuidados em saúde de usuários do SUS ${ }^{2-4}$. Desta forma, é importante a interação de estudantes do curso de Odontologia com estes trabalhadores para enriquecer a formação em saúde.

No processo de construção do vínculo entre serviço e comunidade, um aspecto fundamental é a presença de pressupostos e ações da APS conjuntamente com ações de educação permanente, proporcionando conhecimento multidisciplinar do profissional atuante na rede. Neste caso, o aprendizado de um estudante de Odontologia na perspectiva da APS e a capacitação de ACS para ações de saúde bucal coletiva mostram-se basilares e fundamentais. De acordo com Paulo Freire (1996, 2009) $)^{5,6}$, a formação profissional é enaltecida quando o estudante tem contato 
com meios distintos de aprendizado. Tal processo está associado com o fato de aprender enquanto ensina. $\mathrm{O}$ estudante pode participar de ações de extensão, de forma de disseminar o conhecimento científico na comunidade, bem como enriquecer $o$ aprendizado dos profissionais da rede ${ }^{7-9}$.

$\mathrm{Na}$ perspectiva do ensino em ambientes distintos e considerando a importância da APS na formação de futuros cirurgiões-dentistas, a Faculdade de Odontologia da Universidade Federal do Rio Grande do Sul (FO-UFRGS), em parceria com o Grupo Hospitalar Conceição (GHC), oferta campos de estágios curriculares extramuros em Unidades de Saúde da Família no Município de Porto Alegre, RS, para os alunos do $9^{\circ}$. semestre, com o intuito de proporcionar-lhes experiências em saúde coletiva. Os alunos realizam atividades odontológicas assistenciais, bem como participam de ações de promoção de saúde ${ }^{10}$. Dentro do escopo da educação em saúde, os estudantes têm a possibilidade de participar ativamente no desenvolvimento de cursos de capacitação de ACS, em conjunto com os profissionais das equipes de saúde bucal das Unidades Básicas de Saúde (UBS) ${ }^{3}$.

As atividades de educação em saúde direcionadas aos ACS, por meio de metodologias participativas e aulas expositivas, podem contribuir para fortalecer a capacidade da população no enfrentamento dos problemas de saúde ${ }^{11}$. Agentes comunitários bem preparados e capacitados são capazes de construir vínculos com a comunidade e favorecer o diálogo com os usuários, proporcionando ações de controle e busca ativa de casos de doença. Assim, a atuação do ACS, o qual exerce seu trabalho bem próximo da população, pode contribuir para aumentar o autocuidado das comunidades no controle de determinantes de saúde, ajudando a equipe de saúde bucal na identificação das famílias mais vulneráveis que necessitam de ações mais específicas e na melhora do acesso e da utilização dos serviços básicos de saúde, evitando a assistência odontológica tardia $^{12}$.

As Diretrizes Curriculares Nacionais (DCN) apontam a necessidade de contemplar a formação de cirurgiões-dentistas compromissados, também, com outros modelos assistenciais em saúde, como a Estratégia de Saúde da Família. Para tanto, nesta nova perspectiva de formação em saúde, experiências extramuros vivenciando a realidade das ações de educação em saúde de trabalhadores da APS mostram-se como uma importante ferramenta na formação de profissionais da saúde envolvidos com as comunidades em que atuam.

O objetivo do presente trabalho é apresentar a perspectiva de um estudante da FO-UFRGS sobre sua participação direta nas ações de capacitação de ACS de uma UBS do GHC, durante sua formação.

\section{RELATO DE EXPERIÊNCIA}

Os dados foram coletados por meio de diários de campo e memorandos construídos por meio de observação do participante. $\mathrm{O}$ cenário de pesquisa foi o processo de acontecimento de um curso de capacitação sobre saúde bucal, construído e aplicado por um estudante de Odontologia.

$\mathrm{O}$ aluno inicialmente buscou problematizar a situação de desconhecimento dos ACS sobre cuidados com a saúde bucal na Unidade de Saúde da Família em que estagiava durante o ano de 2014. Nessa problematização identificou a necessidade de ofertar aos referidos agentes uma capacitação sobre o tema. Em um segundo momento construiu uma ação de intervenção, planejando o referido curso. Posteriormente, a ação foi executada na forma de um curso de curta duração dentro da unidade, para todos os seus ACS.

Desse modo, o estudante identificou o primeiro problema como a dificuldade das ACS nos cuidados relacionados à saúde bucal e o segundo como a dificuldade de esclarecer dúvidas das famílias que acompanhavam. $\mathrm{Na}$ ação de capacitação optou-se por encontros contendo apresentações que abordaram 
aspectos sobre a cavidade bucal, demonstração de como instruir higiene bucal e roda de conversa sobre dúvidas quanto a ações em grupos populacionais e linhas de cuidado.

De maneira operacional, estabeleceram-se dois encontros de quatro horas cada um, em que os ACS receberam um portfólio elaborado pela equipe de Saúde Bucal da UBS, como material base e por meio do qual poderia haver interatividade com a apresentação, demonstrações práticas e visuais em exame clínico realizado em um dos integrantes da equipe. Foram abordadas questões diversas de saúde bucal, as quais foram coletadas pelas ACS em grupos populacionais de diferentes condições e/ou morbidades, como gestantes, diabéticos, tabagistas, dentre outros, com a possibilidade de contatos de acordo com a demanda de dúvidas e nível de interesse do grupo.

Para os encontros utilizou-se uma sala de reuniões da unidade e um televisor grande para apresentação de diapositivos. Organizaram-se as cadeiras em semicírculo e, ao centro, foram disponibilizados café, chá e bolos, para descontração da atividade. Estes pequenos detalhes tornam o ambiente mais acolhedor, para que aquele encontro não fosse armazenado na memória dos ACS como algo cansativo ou intimidador, assim como permitir que se sentissem à vontade para expor suas dúvidas e experiências.

$\mathrm{Na}$ percepção do aluno a experiência efetiva pode ser estruturada em momentos. $\mathrm{Na}$ percepção do aluno houve interesse dos ACS em prosseguir com mais encontros. Foi tão enriquecedor para a equipe que, mesmo após o término do estágio, o espaço de construção de conhecimento foi instituído na unidade. No segundo encontro manteve-se o interesse dos ACS, superando as expectativas do aluno tanto na interação durante a capacitação como na continuidade para a realização de um terceiro encontro.

No processo de avaliação da capacitação, o aluno observou inicialmente que os encontros despertaram o interesse dos
ACS em buscar consultas odontológicas para averiguar as suas próprias condições de saúde bucal. De fato, posteriormente ao fechamento da capacitação houve a procura das ACS por atendimentos odontológicos assistenciais.

$\mathrm{Na}$ análise do aluno, frente à experiência vivida, é fundamental observar as demandas odontológicas da população, os programas de saúde realizados na unidade, bem como as dúvidas dos ACS sobre cuidados com saúde bucal para elaboração de uma capacitação, como a apresentada neste relato. Em estudo de Cardoso et al. (2015) $)^{13}$, a experimentação interdisciplinar e humanizada do trabalho em imersão na comunidade é uma experiência enriquecedora e única para a formação do aluno, contribuindo também para melhorar as condições de saúde da comunidade.

O ACS é o primeiro contato da população com a UBS. O aluno, na sua perspectiva se questionou: "caso o ACS não esteja capacitado e informado sobre tais situações (demandas de saúde bucal), como poderiam esclarecer as dúvidas da população? E, com isso, garantir a maior adesão da população aos projetos propostos e ao autocuidado com a saúde bucal?" Portanto, existiam diferenças quanto a questões relativas ao conhecimento de saúde bucal entre os ACS antes e depois da capacitação. Além deste aspecto, houve aumento do número de escovas dentais nas famílias após a capacitação. Os ACS observaram e relataram, durante o último encontro, perceber mais relatos de aumento da frequência da escovação e do uso do fio dental. Neste contexto, as equipes de saúde bucal, juntamente com ACS, refletem uma melhora no acesso e uso mais regular dos serviços pela população, principalmente entre as mulheres ${ }^{14}$.

Em ambiente com tantas demandas como o de uma UBS, pensar as necessidades da população pode parecer algo complexo, afinal são tantos projetos, grupos, sistemas de acolhimento, integração, que o ponto fraco no sistema muitas vezes não é tão óbvio. Cardoso 
et al. $(2015)^{13}$ apontam em sua experiência que ações de alunos em comunidades distintas mostram-se como um estímulo à prática interdisciplinar e multiprofissional e tal processo reflete-se na formação de um profissional ético e consciente do seu papel como cidadão. A interação com a equipe é importante, pois no caso apresentado, a sugestão de que fosse elaborado um curso de capacitação surgiu do enfermeiro da unidade, o qual havia sido questionado pelos ACS sobre dúvidas em relação à saúde bucal trazidas pela comunidade.

Outras dúvidas emergentes ao longo da capacitação eram, na grande maioria, referentes a questões dos projetos existentes para conclusão de metas de consultas odontológicas para diabéticos, crianças até 24 meses e gestantes. O estudante observou que o programa de captação desses grupos populacionais se restringia a telefonar para agendar consulta odontológica. Entretanto, os indivíduos desses grupos passaram a indagar os ACS sobre questões como: "Por que tenho que ir ao dentista se não tenho mais dentes?" "Meus dentes estão bons, por que tenho de ir se sou gestante?" "Meu filho nem tem dentes ainda, porquê tenho de levá-lo ao dentista?" Estas questões despertaram a identificação de nós críticos na informação à população, os quais foram trabalhados na capacitação construída e aplicada pelo aluno de graduação idealizador e relator da presente experiência.

\section{CONSIDERAÇÕES FINAIS}

O trabalho dos ACS é de grande importância para o SUS na busca pelo sucesso das metas propostas nas unidades. Entretanto, para serem realmente efetivos nesse apoio, os agentes necessitam de capacitações. Quando planejadas com a participação de alunos de graduação, podem se constituir também em um processo de aprendizagem para o estudante, associado à capacitação de profissionais de saúde, que surte resultados efetivos para a promoção da saúde e informação da comunidade ${ }^{9}$. Considera-se também a importância das faculdades de
Odontologia que se propõem a fazer parcerias com o SUS para a formação de seus alunos. No presente relato, esta parceria propiciou novos campos de saberes e conhecimentos para o ensino da Odontologia.

\section{ABSTRACT \\ The Dental student's perspective regarding Community Health Agent capacitation}

Currently, the curriculum guidelines for undergraduate Dental curses in Brazil ought to offer opportunities, in different learning scenarios, to a formation that will reflect holistic and integrative perceptions to the health care. This paper presents a case report of an undergraduate dental student experience in the construction of a training course on oral health care for community health workers. This training course was fundamental to the student learning process and to the Family Health Unit workers.

Descriptors: Health Education. Public Health. Unified Health System.

\section{REFERÊNCIAS}

1. Brasil, Ministério da Saúde. Portaria $\mathbf{N}^{\circ}$ 2.488 , de 21 de outubro de 2011. Acesso em: 23 mar 2015. Disponível em: http://bvsms.saude.gov.br/bvs/saudelegis /gm/2011/prt2488_21_10_2011.html.

2. Silva JA. O agente comunitário de saúde do Projeto Qualis: agente institucional ou agente da comunidade? [Tese] São Paulo: Faculdade de Saúde Pública da USP; 2001

3. Kluthcovsky ACGC, Takayanagui AMM. Community health agent: a literature review. Rev Lat Am Enfermagem. 2006;14(6):957-63.

4. Costa ICC, Unfer B, Oliveira AGRC, Arcieri RM, Moraes E, Saliba NA. Investindo na promoção de saúde: PSF e PACS, uma análise de suas performances em oito municípios brasileiros. Rev ABOPREV. 1998;1(1):30-7.

5. Freire P. Pedagogia da autonomia, saberes necessários à prática docente. 19ed. São Paulo: Paz e Terra, 1996. 
6. Freire P. Educação como prática da liberdade. Rio de Janeiro: Paz e Terra, 2009

7. Silva MS, Vasconcelos SD. Extensão Universitária e formação profissional: avaliação da experiência das Ciências Biológicas na Universidade Federal de Pernambuco. Est Aval Educ. 2006;17(33):119-36.

8. Paulino VCP, Bezerra ALQ, Branquinho NCDSS, Paranaguá TDB. Ações de educação permanente no contexto da estratégia saúde da família. Rev Enferm UERJ. 2012;20(3):368-73.

9. Weigelt D, Weigelt ID, Rezendo MS, Schilling AZ, Krug SBF. A comunicação, a educação no processo de trabalho e o cuidado na rede pública de saúde do Rio Grande do Sul: cenários e desafios. Rev Eletr Comunic Inf Inov Saúde. 2015;9(3):1-8.

10. Schonhofen A, Plegge J, Warmiling CM, Scalco G, Santos JA, Oliveira P, Bulgarelli AF. Projeto Terapêutico Singular no processo ensinoaprendizagem de alunos em estágio supervisionado: relato de uma experiência efetiva. Rev ABENO. 2010; 10(2):59-63.
11. Bastos ACS. O impacto sociocultural do Programa de Saúde da Família (PSF): uma proposta de avaliação. Cad Saúde Pública. 1998;14(2):429-35.

12. Brizolara RV, Oliveira AC, Frazão P. Problemas de saúde bucal coletiva: percepções de agentes comunitários de saúde da região do $\mathrm{ABC}$, Estado de São Paulo, Brasil. Cienc Saude Coletiva. 2003;8(Supl2):104.

13. Cardoso AC, Corralo DJ, Krahl M, Alves LP. O estimulo à prática da interdisciplinaridade e do multiprofissionalismo: a Extensão Universitária como uma estratégia para a educação interprofissional. Rev ABENO. 2015;15(2):12-9.

14. Frazão P, Marques D. Efetividade de programa de agentes comunitários na promoção da saúde bucal. Rev Saúde Públ. 2009;43(3):463-471.

Correspondência para:

Alexandre Favero Bulgarelli

e-mail: alexandre.bulgarelli@ufrgs.br

Faculdade de Odontologia da UFRGS

Rua Ramiro Barcelos, 2492

90035-004, Porto Alegre - RS 